\title{
ESTRUTURA ESPACIAL ARBÓREA DE UM REMANESCENTE NATURAL DE FLORESTA OMBRÓFILA MISTA
}

\author{
Allan Libanio Pelissari ${ }^{*}$, Afonso Figueiredo Filho², Carlos Roberto Sanquetta ${ }^{3}$, Angelo Augusto \\ Ebling ${ }^{4}$, Marcelo Roveda ${ }^{5}$, Vinicius Costa Cysneiros ${ }^{6}$ \\ 1* Universidade Federal do Paraná, Departamento de Ciências Florestais, Curitiba, Paraná, Brasil, allanpelissari@gmail.com \\ ${ }^{2}$ Universidade Estadual do Centro-Oeste, Departamento de Engenharia Florestal, Irati, Paraná, Brasil, afigfilho@gmail.com \\ ${ }^{3}$ Universidade Federal do Paraná, Departamento de Ciências Florestais, Curitiba, Paraná, Brasil, \\ carlos sanquetta@hotmail.com \\ ${ }^{4}$ Universidade Federal Rural da Amazônia, Departamento de Engenharia Florestal, Parauapebas, Pará, Brasil, \\ aebling@hotmail.com \\ ${ }^{5}$ Universidade Federal do Paraná, Pós-graduação em Engenharia Florestal, Curitiba, Paraná, Brasil, \\ marcelo roveda@hotmail.com \\ ${ }^{6}$ Universidade Federal do Paraná, Pós-graduação em Engenharia Florestal, Curitiba, Paraná, Brasil, \\ vccysneiros.florestal@gmail.com
}

\begin{abstract}
Resumo
O desconhecimento dos padrões ecológicos nas florestas naturais contribui para a busca por ferramentas inovadoras que investiguem suas relações e alterações espaciais. Para isso, atualmente, a geoestatística destaca-se como um conjunto de análises que possibilita a estimativa estatisticamente adequada de uma variável e o seu mapeamento. Dessa forma, o presente trabalho objetivou modelar, mapear e relacionar os padrões e dinâmicas espaciais do estoque em área basal dos estratos arbóreos: inferior, médio e superior, de um remanescente de Floresta Ombrófila Mista na região Sul do Brasil. As informações da composição florística foram obtidas por meio de um inventário contínuo executado em 400 unidades georreferenciadas de $25 \mathrm{~m} \times 25 \mathrm{~m}$ e mensuradas em 2002, 2008 e 2014. Posteriormente, a geoestatística foi utilizada para modelar os padrões espaciais e a krigagem ordinária pontual foi aplicada para compor os mapas temáticos. Os resultados corroboraram com a hipótese de que a estrutura da floresta apresenta dependência espacial e distribuição heterogênea ao longo dos levantamentos, cujos comportamentos espaciais dos estratos arbóreos estiveram relacionados às dimensões diamétricas dos indivíduos e à diversidade de espécies.
\end{abstract}

Palavras-chave: Floresta Ombrófila Mista; Variabilidade espacial; Geoestatística.

\begin{abstract}
Tree spatial structure of a native remnant of Araucaria Forest. The lack of ecological patterns in natural forests contributes to the search for innovative tools to investigate their relations and spatial changes. For this reason, currently, the geostatistical analysis stands out as a set of analyses that enable statistically suitable estimate of a variable and its mapping. Thus, the present work aimed to shape, mapping and relate the patterns and spatial dynamics of stock of basal area in ecological strata: lower, middle and upper of a native remnant of Araucaria Forest in the South region of Brazil. The information of the floristic composition was obtained by means of a continuous inventory runs on 400 georeferenced units of $25 \mathrm{~m} \times 25 \mathrm{~m}$ in the years of 2002, 2008 e 2014. Subsequently, the geostatistical analysis was used to model the spatial patterns and the punctual ordinary kriging and was applied to compose the thematic maps. The results corroborate with the hypothesis that the structure of the forest presents spatial dependence and heterogeneous distribution along the surveys, whose spatial behavior of the strata were related to the diameter dimensions of the trees and to species diversity.
\end{abstract}

Keywords: Mixed Ombrophilous Forest; Spatial variability; Geostatistics. 


\section{INTRODUÇÃO}

Durante o processo de ocupação da região Sul do Brasil, significativa pressão antrópica foi deferida sobre as extensas áreas de Floresta Ombrófila Mista, resultando em intensa exploração madeireira e substituição da vegetação natural por pastagens e culturas agrícolas. Esse processo, iniciado no princípio do século $X X$, acarretou na redução drástica das áreas naturais para atuais $1 \%$ ocupados por remanescentes florestais (SANQUETTA et al., 2003; MEDEIROS et al., 2005).

Essa desordenada exploração, principalmente dos indivíduos arbóreos de elevado valor econômico, tornou a Araucaria angustifolia (Bertol.) Kuntze uma espécie em relevante vulnerabilidade na flora brasileira. Todavia, atualmente os remanescentes de Florestas Ombrófilas Mistas ainda sofrem a pressão da expansão das monoculturas, o que agrava o desmatamento nos pequenos fragmentos e nas bordas dos principais remanescentes (MEDEIROS et al., 2005).

Posto que o desconhecimento dos padrões ecológicos e as significativas variações existentes ao longo das áreas de vegetação natural de Floresta Ombrófila Mista contribuem para a definição de critérios subjetivos para a conservação dos recursos florestais, amplia-se a busca por ferramentas inovadoras que investiguem os fatores que determinam o comportamento da estrutura arbórea e da sucessão ecológica. Essa necessidade decorre do fato de que a dificuldade na mensuração da estrutura espacial das florestas esconde, frequentemente, a real variabilidade existente no espaço populacional.

Para isso, atualmente a geoestatística destaca-se como um conjunto de técnicas estatísticas e matemáticas que permite a modelagem de uma determinada variável para o seu posterior mapeamento. Assim, por meio da teoria das variáveis regionalizadas (MATHERON, 1971), considerada a fundamentação teórica da geoestatística, uma função espacial numérica de um fenômeno estruturado no espaço possibilita mensurar as taxas de mudanças de uma variável regionalizada e, via interpolador geoestatístico, estimá-la nos locais não amostrados (DAVIS, 2002; WEBSTER; OLIVER, 2007).

Dessa forma, considerando a hipótese de que a estrutura das florestas naturais apresenta dependência espacial mensurável para mapeamentos estatisticamente precisos de sua complexidade e das alterações temporais, o presente trabalho objetivou modelar, mapear e relacionar o padrão e a dinâmica espacial do estoque em área basal dos estratos arbóreos em um remanescente de Floresta Ombrófila Mista na região Sul do Brasil.

\section{MATERIAL E MÉTODOS}

Os dados foram provenientes de um inventário florestal contínuo, executado nos anos de 2002, 2008 e 2014, em um remanescente de Floresta Ombrófila Mista localizado na Unidade de Conservação da Floresta Nacional de Irati, Sul do Brasil, entre as coordenadas $25^{\circ} 01^{\prime} \mathrm{S}$ e $25^{\circ} 40^{\prime} \mathrm{S}$ e $51^{\circ} 11^{\prime} \mathrm{W}$ e $51^{\circ} 15^{\prime} \mathrm{W}$. A estrutura vertical da floresta foi estratificada pela altura da copa das árvores e pela sua capacidade de interceptar a radiação luminosa, resultando nos estratos: 1) inferior, sem iluminação direta sobre a copa; 2) médio, com iluminação superior parcial; e 3) superior, com iluminação completa.

Os resultados do estoque em área basal dos estratos foram submetidos à análise estatística descritiva e ao teste de Kolmogorov-Smirnov ao nível de 95\% de probabilidade (FELDMAN; VALDEZFLORES, 2010). Posteriormente, os padrões espaciais foram modelados por meio da análise geoestatística, considerando o cálculo das semivariâncias (1), o posicionamento geográfico central de cada unidade amostral $(x, y)$ e o posterior cômputo das distâncias $(h)$ e das diferenças numéricas das variáveis $(Z)$ na malha de pontos.

$$
\gamma(h)=\frac{1}{2 N(h)} \sum_{i=1}^{N(h)}\left\{\left[Z\left(x_{i}+h\right)-Z\left(x_{i}\right)\right]^{2}\right\}
$$

Em que: $y(h)=$ semivariância de $Z\left(x_{i}\right) ; h=$ distância; e $N(h)=$ número de pares de pontos medidos $Z\left(x_{i}\right)$ e $Z\left(x_{i}+h\right)$, separados por uma distância $h$.

As semivariâncias foram determinadas entre os pontos amostrais equidistantes, cujo processo foi repetido em quatro direções no plano espacial: $0^{\circ}, 45^{\circ}, 90^{\circ}$ e $135^{\circ}$, dos quais foi obtida a matriz das semivariâncias médias entre as distâncias equivalentes. Assim, para as estimativas das semivariâncias em quaisquer distâncias entre as unidades amostrais, foram ajustados os modelos de semivariogramas: Esférico (2), Exponencial (3) e Gaussiano (4), com o auxílio do programa computacional GS+ (ROBERTSON, 2008) e de planilhas eletrônicas. 
A avaliação e a seleção dos melhores ajustes foram baseadas na menor soma de quadrados dos desvios ponderados (SQDP) e no maior coeficiente de determinação $\left(R^{2}\right)$. Ao passo que a interpolação e a espacialização foram efetuadas por meio da krigagem ordinária pontual (WEBSTER; OLIVER, 2007). Posteriormente, os mapas temáticos foram produzidos com o programa computacional Surfer (GOLDEN SOFTWARE, 2002), considerando cinco classes com intervalos relativos de $20 \%$ da amplitude observada.

$$
\begin{aligned}
& \gamma(h)=\left\{\begin{array}{l}
C_{0}+C\left[\left(\frac{3}{2}\right)\left(\frac{h}{a}\right)-\left(\frac{1}{2}\right)\left(\frac{h}{a}\right)^{3}\right] \\
C_{0}+C
\end{array} \begin{array}{r}
\text { se } h \leq a \\
\text { se } h>a
\end{array}\right. \\
& \gamma(h)=C_{0}+C\left(1-e^{-h / a}\right) \\
& \gamma(h)=C_{0}+C\left(1-e^{-h^{2} / a^{2}}\right)
\end{aligned}
$$

Em que: $\gamma(h)=$ semivariância de $Z\left(x_{i}\right) ; h=$ distância; $C_{0}=$ efeito pepita; $C=$ variância a priori dos dados; $C_{0}+C=$ patamar; e $a=$ alcance.

\section{RESULTADOS E DISCUSSÃO}

Por meio do inventário florestal contínuo executado no remanescente de Floresta Ombrófila Mista, 124 espécies arbóreas foram identificadas em 46 famílias botânicas, com destaque de importância para a Araucaria angustifolia (Bertol.) Kuntze, como a espécie de maior área basal para o estrato superior; seguida pela Ocotea odorifera Rohwer no estrato médio; e Coussarea contracta (Walp.) Müll. Arg. no estrato inferior.

O estrato inferior apresentou os menores valores médios em área basal $(\bar{G})$, com coeficiente de variação (CV) superior a 100\% (Tabela 1), ao passo que as maiores áreas basais e seus incrementos periódicos (IPA) foram identificados no estrato superior, com coeficientes de variação inferiores a $50 \%$. De modo geral, a tendência numericamente crescente das médias e decrescente das variabilidades foi observada entre as ocasiões dos levantamentos, com normalidade apenas para o total, estrato superior e secundárias iniciais, avaliados pelo teste $K S$.

Tabela 1. Análise estatística descritiva do estoque em área basal dos estratos arbóreos de um remanescente de Floresta Ombrófila Mista

\begin{tabular}{ccccccccccc}
\hline \multirow{2}{*}{ Área basal } & \multicolumn{3}{c}{$\mathbf{2 0 0 2}$} & \multicolumn{3}{c}{$\mathbf{2 0 0 8}$} & \multicolumn{2}{c}{$\mathbf{2 0 1 4}$} & \multirow{2}{*}{ IPA } \\
\cline { 2 - 10 } & $\bar{G}$ & $C V$ & $K S$ & $\bar{G}$ & $C V$ & $K S$ & $\bar{G}$ & $C V$ & $K S$ & \\
\hline Inferior & 0,77 & $114,9 \%$ & $0,192^{*}$ & 1,11 & $89,6 \%$ & $0,132^{*}$ & 1,43 & $77,9 \%$ & $0,106^{*}$ & 0,06 \\
Médio & 11,02 & $58,5 \%$ & $0,091^{*}$ & 11,30 & $56,0 \%$ & $0,103^{*}$ & 11,78 & $52,6 \%$ & $0,095^{*}$ & 0,06 \\
Superior & 16,89 & $45,8 \%$ & $0,051^{\text {ns }}$ & 17,80 & $45,8 \%$ & $0,051^{\text {ns }}$ & 18,32 & $46,5 \%$ & $0,056^{\text {ns }}$ & 0,12 \\
\hline
\end{tabular}

Em que: $\bar{G}=$ média da área basal $\left(\mathrm{m}^{2} \mathrm{ha}^{-1}\right) ; C V=$ coeficiente de variação em porcentagem; $K S=$ teste de Kolmogorov-Smirnov; IPA = incremento periódico anual em área basal $\left(\mathrm{m}^{2} \mathrm{ha}^{-1} \mathrm{ano}^{-1}\right)$ entre os anos de $2002 \mathrm{e}$ 2014; ns = há distribuição normal; $\mathrm{e}^{*}=$ não há distribuição normal a 5\% de significância.

Posteriormente, na Figura 1 estão apresentados os parâmetros dos modelos geoestatísticos ajustados, em que houve predominância do modelo Esférico, cujos valores de alcance (a) expressaram a distância máxima em que dois pontos amostrais correlacionaram-se espacialmente. Ademais, por meio dos semivariogramas selecionados (Figura 1), foi verificado o efeito crescente das semivariâncias ao longo da distância, partindo do efeito pepita até atingir um valor estável, identificado como o patamar.

Os ajustes dos semivariogramas selecionados resultaram nas menores somas de quadrados dos desvios ponderados (SQDP) e nos maiores coeficientes de determinação $\left(R^{2}\right)$, sendo superiores a 0,89 para os estratos inferiores (Figuras 1A até 1C) e médios (Figuras 1D até 1F); enquanto os valores de $R^{2}$ entre 0,78 e 0,83 para o estrato superior (Figuras $1 G$ até 1 I) decorreram da maior dispersão das semivariâncias observadas em torno da linha média estimada.

Com base nos mapas temáticos (Figura 2), padrões espaciais distintos foram verificados, em que a principal dinâmica foi observada com a redução da área da classe I do estrato inferior nos intervalos de 1,2 a 2,4 $\mathrm{m}^{2}$ ha-1 (Figuras 2A até 2C); enquanto ganho representativo pela classe II, entre 
7,2 e $14,4 \mathrm{~m}^{2}$ ha-1 , foi verificado para o estrato médio na porção esquerda do fragmento florestal (Figuras 2D até 2F). Contudo, a maior estabilidade da área basal entre as ocasiões foi observada no estrato superior (Figuras $2 \mathrm{G}$ até 2l). Nesses casos, as classes superiores de área basal foram representadas por pequenos fragmentos, ao passo que a maior semelhança espacial foi identificada na região centro-superior.

(A) Estrato inferior - 2002

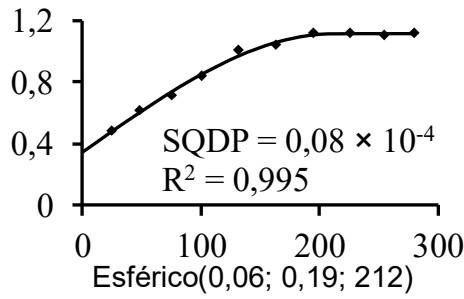

(D) Estrato médio - 2002

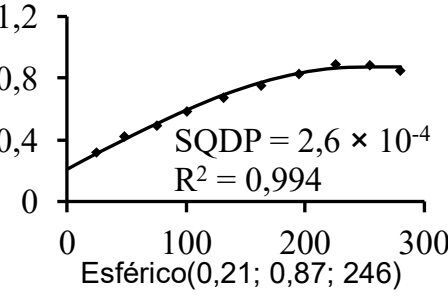

(G) Estrato superior - 2002

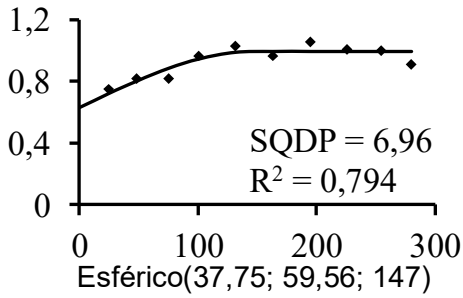

(B) Estrato inferior - 2008

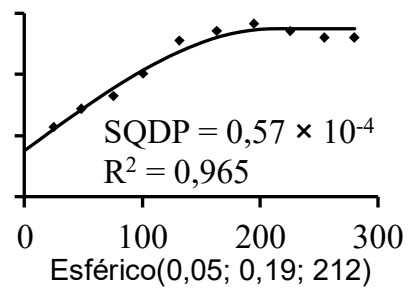

(E) Estrato médio - 2008

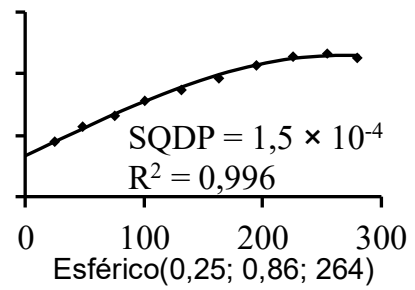

(H) Estrato superior - 2008

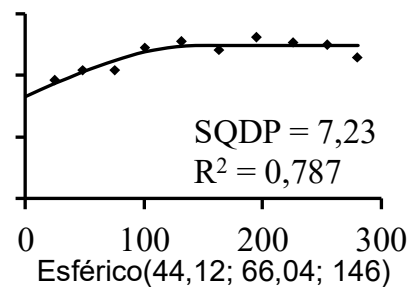

Distância (m)
(C) Estrato inferior - 2014

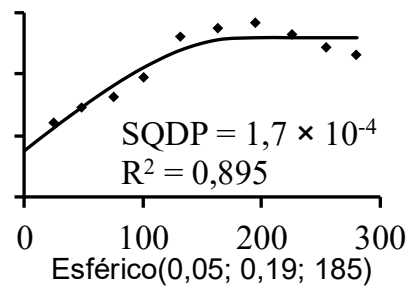

(F) Estrato médio - 2014

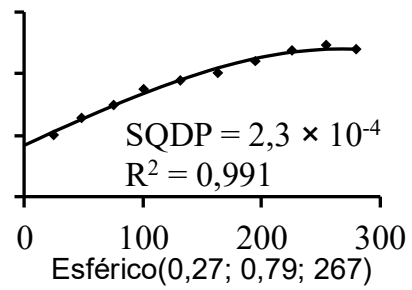

(I) Estrato superior - 2014

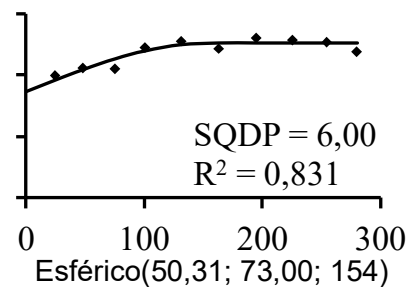

Figura 1. Semivariogramas selecionados para o estoque em área basal dos estratos arbóreos de um remanescente de Floresta Ombrófila Mista. Em que: SQDP = soma de quadrado dos desvios ponderados; $\mathrm{R}^{2}=$ coeficiente de determinação; e $\operatorname{Modelo}\left(C_{0} ; C_{0}+C_{1} ; a\right)$.

Os resultados do presente trabalho corroboraram a hipótese de que a estrutura da Floresta Ombrófila Mista apresenta dependência espacial, cujos padrões e dinâmicas na espacialização da área basal dos estratos arbóreos foram modelados e mapeados em três ocasiões do inventário contínuo. Com isso, essas considerações permitiram observar valores diferenciados no estoque e no incremento em área basal do remanescente florestal (Tabela 1), possibilitando visualizar gradientes de distribuição heterogênea da estrutura vertical (Figuras 2).

Nas ocasiões do inventário contínuo (Tabela 1), o estrato superior representou aproximadamente $59 \%$ da área basal do remanescente florestal, seguido por $38 \%$ para o estrato médio e de 3\% para o inferior. Isso indicou a existência de uma relação direta entre a estrutura vertical e a área basal, em que indivíduos com maior diâmetro estão situados nos estratos superiores. Ademais, a maior homogeneidade na distribuição espacial do estrato superior (Figuras 2G a 2I) resultou da predominância de Araucaria angustifolia, ao passo que a maior diversidade arbórea nos estratos inferior e médio, em torno de 112 e 122 espécies, respectivamente, além do ingresso de indivíduos de Coussarea contracta e de Ocotea odorifera ao longo do inventário contínuo nesses estratos, resultaram na maior variabilidade (Tabela 1) e na heterogeneidade espacial (Figuras 2A até 2F). 
(A) Estrato inferior - 2002

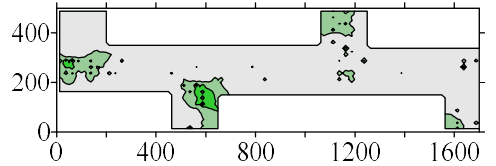

(D) Estrato médio - 2002

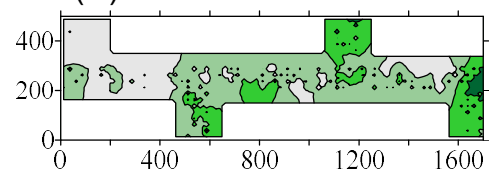

(G) Estrato superior - 2002

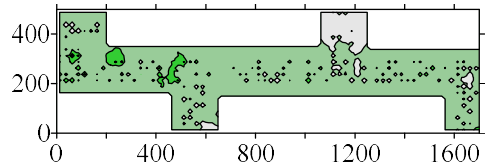

(B) Estrato inferior - 2008

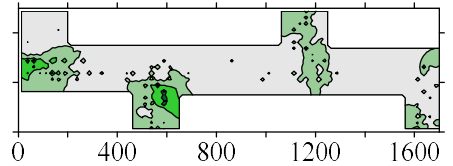

(E) Estrato médio - 2008

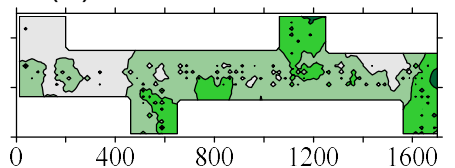

(H) Estrato superior - 2008

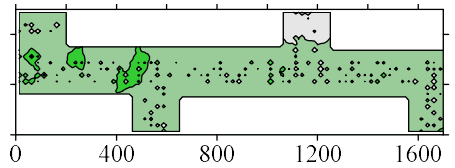

\begin{tabular}{|c|c|cccc}
\multicolumn{1}{|c}{ I } & \multicolumn{1}{c}{ II } & III & IV & V \\
\hline & & & & & \\
0 & 20 & 40 & 60 & 80 & $100 \%$
\end{tabular}

Percentual da área basal
(C) Estrato inferior - 2014

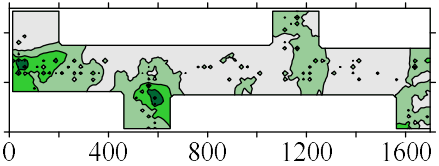

(F) Estrato médio - 2014

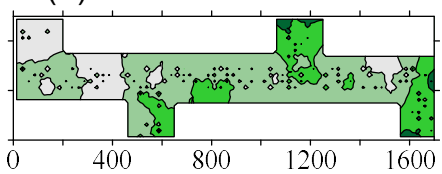

(I) Estrato superior - 2014
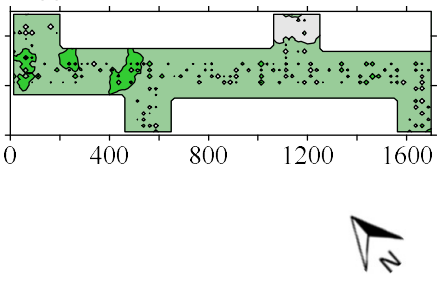

Figura 2. Mapas temáticos do estoque em área basal dos estratos arbóreos de um remanescente de Floresta Ombrófila Mista.

A ocorrência de algumas espécies em todos os estratos arbóreos, como é comumente observado com a Araucaria angustifolia (SOUZA, 2007), dificulta relacionar a distribuição espacial da estrutura vertical com a ocorrência de determinadas espécies. Contudo, os indivíduos com menores diâmetros contribuíram pouco para a formação do estoque em área basal no estrato inferior, cuja presença constitui uma reserva estratégica para a manutenção da capacidade regenerativa da floresta (RIBEIRO et al., 2013). Além disso, a formação de pequenas clareiras naturais constituiu um potencial fator na dinâmica espacial dos estratos inferior (Figuras 2A até $2 \mathrm{C}$ ) e médio (Figuras 2D até 2F), ao reduzir a dominância de algumas espécies e ao aumentar a disponibilidade de recursos vitais ao desenvolvimento dos indivíduos emergentes (OKUDA et al., 1997; WIEGAND et al., 1998; FUCHS et al., 2013; ZHU et al., 2013; NGUYEN et al., 2014).

\section{CONCLUSÕES}

A estrutura da Floresta Ombrófila Mista apresenta dependência espacial modelável pelas análises geoestatísticas, o que possibilita obter mapeamentos estatisticamente precisos da complexidade dos padrões e das dinâmicas espaciais da área basal dos estratos arbóreos no decorrer do inventário florestal contínuo.

O remanescente florestal é composto por um mosaico de padrões espaciais heterogêneos, cujas características espaciais dos estratos arbóreos estão diretamente relacionadas com as dimensões diamétricas dos indivíduos e com a diversidade de espécies ao longo da estrutura vertical, ao passo que a dinâmica espacial na floresta é influenciada pelas taxas de incremento em área basal detectadas nas diferentes ocasiões de realização do inventário florestal contínuo.

\section{AGRADECIMENTOS}

O presente trabalho foi realizado com apoio do CNPq, Conselho Nacional de Desenvolvimento Científico e Tecnológico - Brasil.

\section{REFERÊNCIAS}

DAVIS, J. C. Statistic and data analysis in geology. New York: John Wiley \& Sons, 3 ed. 2002.656 p. 
FELDMAN, R. M.; VALDEZ-FLORES, C. Applied probability and stochastic processes. New York: Springer, 2 ed. 2010. 397 p.

FUCHS, E. J.; ROBLES, T.; HAMRICK, J. L. Spatial distribution of Guaiacum sanctum (Zygophyllaceae) seedlings and saplings relative to canopy cover in Palo Verde National Park, Costa Rica. Revista de Biología Tropical, v. 61, n. 3, p. 1521-1533, 2013.

GOLDEN SOFTWARE. Surfer: user's guide. Colorado: Golden Software, 2002. 664 p.

MATHERON, G. The theory of regionalized variables and its applications. Fontainebleau: École Nationale Supérieure des Mines de Paris, 1971. 211 p.

MEDEIROS, J. D.; SAVI, M.; BRITO, B. F. A. Seleção de área para criação de unidade de conservação na Floresta Ombrófila Mista. Biotemas, v. 18, n. 3, p. 33-50, 2005.

NGUYEN, H.; WIEGAND, K.; GETZIN, S. Spatial patterns and demographics of Streblus macrophyllus trees in a tropical evergreen forest, Vietnam. Journal of Tropical Forest Science, v. 26, n. 3, p. 309$319,2014$.

OKUDA, T.; KACHI, N.; YAP, S. K.; MANOKARAN, N. Tree distribution pattern and fate of juveniles in a lowland tropical rain forest - implications for regeneration and maintenance of species diversity. Plant Ecology, v. 131, n. 2, p. 155-171, 1997.

RIBEIRO, T. M.; IVANAUSKAS, N. M.; MARTINS, S. V.; POLISEL, R. T.; SANTOS, R. L. R.; MIRANDA NETO, A. Mixed rain forest in southeastern Brazil: tree species regeneration and floristic relationships in a remaining stretch of forest near the city of Itaberá, Brazil. Acta Botanica Brasilica, v. 27, n. 1, p. 71-86, 2013

ROBERTSON, G. P. GS+: Geostatistics for the environmental sciences. Plainwell: Gamma Design Software, 2008. 179 p.

SANQUETTA, C. R.; DALLA CORTE, A. P.; EISFELD, R. L. Crescimento, mortalidade e recrutamento em duas florestas de Araucária (Araucaria angustifolia (Bert.) O. Ktze.) no Estado do Paraná, Brasil. Revista Ciências Exatas e Naturais, v. 5, n. 1, p. 101-112, 2003.

SOUZA, A. F. Ecological interpretation of multiple population size structures in trees: the case of Araucaria angustifolia in South America. Austral Ecology, v. 32, p. 524-533, 2007.

WEBSTER, R.; OLIVER, M. A. Geostatistics for environmental scientists. West Sussex: John Wiley \& Sons Ltd, 2. ed. 2007. 333 p.

WIEGAND, T.; MOLONEY, K. A.; MILTON, S. J. Population dynamics, disturbance, and pattern evolution: identifying the fundamental scales of organization in a model ecosystem. The American Naturalist, v. 152, n. 3, p. 321-337, 1998.

ZHU, J.; MATSUZAKI, T.; LEE, F. GONDA, Y. Effect of gap size created by thinning on seedling emergency, survival and establishment in a coastal pine forest. Forest Ecology and Management, v. 182, n. 1-3, p. 339-354, 2013. 